\title{
M. Steinhoff Lymphomatoide Papulose - ein Schaf im Wolfspelz
}

\author{
Lymphomatoid Papulosis - A Sheep in Wolf's Skin
}

\section{Zusammenfassung}

Bei der lymphomatoiden Papulose (LyP) handelt es sich um eine CD30-positive T-Zell-lymphoproliferative Erkrankung mit guter Prognose, aber häufig langjährigem, rezidivierendem Verlauf. Bei etwa 10-20\% der Patienten mit LyP wird das Auftreten von malignen Lymphomen beobachtet. Aufgrund der Diskrepanz zwischen klinisch benignem Verlauf und malignem histologischen Zellbild wurde die Frage, ob es sich bei der LyP um eine reaktiv-entzündliche Erkrankung oder um ein niedrigmalignes kutanes T-Zell-Lymphom handelt, lange Zeit kontrovers diskutiert. Hinweise auf einen klonalen Ursprung der LyP erbrachten PCRUntersuchungen der T-Zell-Rezeptorumlagerung in Hautläsionen der LyP und assoziierter maligner Lymphome, die das Vorkommen eines identischen T-Zell-Klons in LyP als auch assoziiertem Lymphom zeigen konnten. Die Vermutung, dass es sich bei der LyP per se um eine monoklonale Erkrankung handelt, ließ sich kürzlich auf Einzelzellebene bestätigen. Dabei wurde gezeigt, dass sich der chronische Verlauf der LyP mit den für diese Erkrankung charakteristischen rezidivierenden, spontan abheilenden Hautläsionen auf die Expansion und Regression eines identischen T-Zell-Klons zurückführen lässt. Dementsprechend wird die LyP sowohl in der EORTC- als auch in der WHO-Klassifikation der Lymphome als niedrigmalignes T-Zell-Lymphom geführt, nimmt jedoch aufgrund des benignen Verlaufs mit einer 10-Jahres-Überlebensrate von 100\% eine Sonderstellung ein.

\section{Abstract}

Lymphomatoid papulosis (LyP) represents a cutaneous CD30-positive T-cell lymphoproliferative disorder that takes a clinical benign course, often over a period of several years. From $10-20 \%$ of LyP cases are associated with malignant lymphoma. Due to the contrast of benign clinical course and malignant histological picture the nosological position of LyP was a long-standing enigma. On the one hand LyP was assigned to the group of benign reactive processes, on the other hand it was seen as a cutaneous lymphoma. By the finding of the same T-cell receptor rearrangements in the LyP as well as in the associated malignant lymphoma, LyP was identified as a precursor lesion. Recently single-cell analysis of T-cell receptor rearrangements in LyP cases being not associated with malignant lymphoma demonstrated that LyP unequivocally represents a monoclonal T-cell disorder. Moreover it was shown that the prolonged course of LyP with its typical features of waxing and waning is due to the expansion and regression of a single T-cell clone. According to these findings, nowadays LyP is included as a cutaneous low grade malignant lymphoma in both the EORTC- and WHO lymphoma classification. However due to its favourable 10 -year survival rate of $100 \%$, LyP occupies an exceptional position in the spectrum of cutaneous lymphoma. 
Die lymphomatoide Papulose (LyP) wurde erstmals 1968 durch Macauly beschrieben [1]. Dabei handelt es sich um eine seltene lymphoproliferative Hauterkrankung mit zumeist benignem Verlauf (Inzidenz 1,2 - 1,9 Fälle/1 000 000) [2,3]. Klinisch charakteristisch ist das rezidivierende Auftreten selbstheilender erythematöser, z.T. zentral nekrotischer Papeln und Knötchen (Abb.1). Trotz des für die Erkrankung typischen Phänomens der spontanen Abheilung individueller Läsionen nimmt die LyP nicht selten einen chronisch-rezidivierenden Verlauf über Jahrzehnte.

Dem benignen klinischen Verlauf steht ein ungewöhnliches histologisches Bild mit Hodgkin-Reed-Sternberg-ähnlichen, atypischen CD30-positiven T-Lymphozyten, eingebettet in ein z.T. stärkeres reaktives entzündliches Infiltrat gegenüber (Abb.2). Die histologischen Merkmale der LyP sind variabel, so dass drei verschiedene Typen der LyP, Typ A, B und C, beschrieben wurden [4]. Eine allein auf histologischen Merkmalen beruhende Abgrenzung der LyP von anderen kutanen Lymphommanifestationen, inbesondere dem kutanen CD30-positiven großzelligen T-Zell-Lymphom, ist schwierig und in manchen Fällen nicht möglich [5].

\section{Lymphom oder reaktiv entzündlicher Prozess}

Aufgrund der beiden widersprüchlichen Merkmale, „klinisch benigner Verlauf“ und „histologisch malignes Zellbild“, warf die LyP lange Zeit Fragen hinsichtlich ihrer nosologischen Zuordnung auf. Die einen hielten sie für einen reaktiven Prozess [6,7], andere für ein niedrigmalignes kutanes Lymphom [8]. Zur Klärung dieser Frage trugen folgende sowohl klinische Beobachtungen als auch histologische und molekularbiologische Untersuchungen bei.

Bei 10-20\% der Patienten mit LyP kommt es zum Auftreten maligner kutaner/nodaler Lymphome. Beschrieben ist die Assoziation mit dem CD30-positiven großzelligen T-Zell-Lymphom, der Mycosis fungoides oder selten dem Hodgkin Lymphom [9-11]. Sowohl immunhistologisch als auch insbesondere durch Nachweis eines identischen T-Zell-Klons in Hautläsionen von LyP und assoziiertem Lymphom ließ sich in vielen dieser Fälle die LyP als „Vorläufer“ des malignen Lymphoms identifizieren [12-15]. Somit ergab sich die Vermutung, dass es sich bei der LyP per se um eine monoklonale T-Zell-Proliferation handelt.

Klonalitätsuntersuchungen von Patienten mit LyP ohne Assoziation mit malignen Lymphomen, die repräsentativ mehr als $80 \%$ der Fälle von LyP darstellen, erbrachten jedoch widersprüchliche Ergebnisse. Eine klonale T-Zell-Population konnte nur in einem Teil der untersuchten Läsionen gefunden werden und zeigte sich zudem in anatomisch unterschiedlichen Läsionen eines Patienten nicht immer identisch [16-19].

Als ursächlich hierfür waren sowohl biologische als auch methodische Erklärungen in Betracht zu ziehen. Einerseits könnte es sich bei der LyP um ein Kontinuum aus einer zunächst poly-/oligoklonalen Erkrankung handeln, die sich in 10-20\% der Fälle zu einer klonalen malignen T-Zell-Proliferation mit möglichem

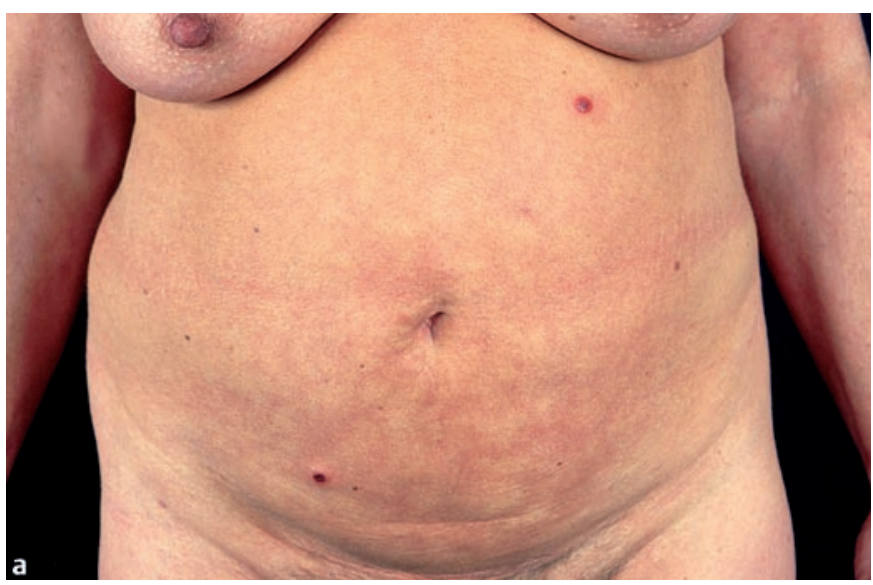

Abb. 1 a Zentral nekrotische, erythematöse Knötchen im Bereich des Abdomens einer Patientin mit LyP.

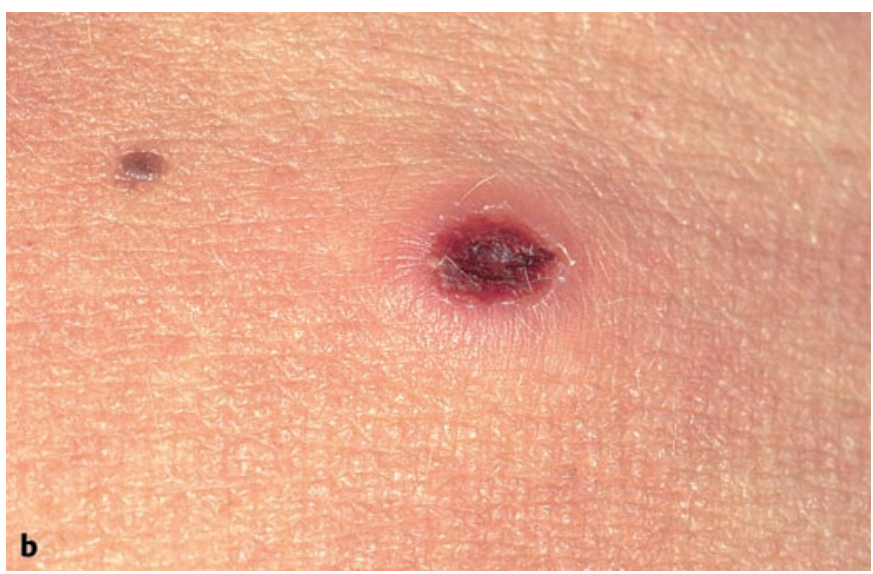

Abb. 1 b Detail

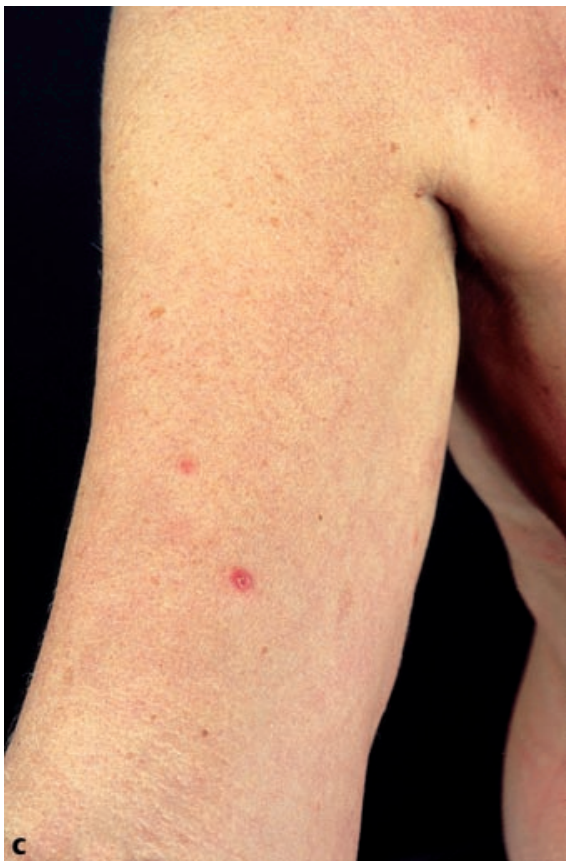

Abb. 1 c Kleine erythematöse Papel im Bereich des rechten Oberarms derselben Patientin. 


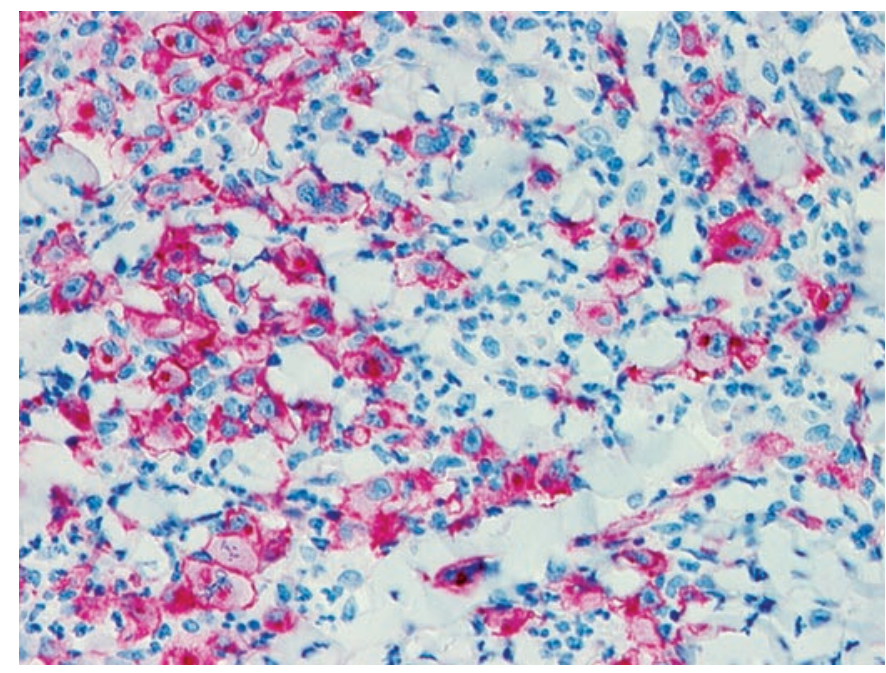

Abb. 2 Immunhistologie (APAAP): CD30-positive, atypische blastäre Zellen sowie stärkeres reaktives entzündliches Infiltrat.

Übergang in ein malignes Lymphom entwickelt. Anderseits wurden in den bis dato vorliegenden Studien Methoden wie Southern blot- oder PCR-Analyse von DNA-Gesamtgewebeextrakt verwendet, die nicht immer ausreichende Sensitivität besitzen, um eine kleine Population klonaler Zellen vor einem polyklonalen Hintergrund, bestehend aus reaktiven Zellen, zu identifizieren. Zudem haben diese Methoden den Nachteil, eine gefundene Klonalität nicht einer histomorphologisch definierten Zellpopulation zuordnen zu können und somit die Frage zu beantworten, ob die klonale Zellpopulation ausschließlich die atypischen CD30-positiven Zellen umfasst oder ob auch morphologisch unauffällige CD30-negative Zellen dem Klon zugehören.

Einzelzellanalyse CD30-positiver und CD30-negativer Zellen der lymphomatoiden Papulose

Um diese Fragen zu klären und dabei erwähnte methodische Probleme zu umgehen, wurde die T-Zell-Rezeptor- $\gamma$-Umlagerung einzelner mikrodissezierter sowohl CD30-positiver als auch CD30-negativer T-Lymphozyten isoliert aus Läsionen von klassischen Patienten mit LyP ohne assoziiertem malignen Lymphom analysiert (Abb. 3) [20]. Diese Untersuchung von 14 Hautbiopsaten von 11 Patienten konnte zeigen, dass die atypischen CD30-positiven Zellen einer Läsion einem Zellklon entstammen. Darüber hinaus ließ sich der identische CD30-positive Zellklon auch in anatomisch und zeitlich (bis zu 52 Monaten) unterschiedlichen Hautläsionen nachweisen [20]. Die untersuchten CD30-negativen Zellen zeigten sich in dieser Studie bis auf wenige Ausnahmen polyklonal und nicht dem CD30-positiven Zellklon zugehörig. Somit ließ sich die auf früheren Studien basierende Vermutung, dass es sich bei der LyP tatsächlich um eine monoklonale CD30-positive T-Zell-Erkrankung handelt, erstmals auf Einzelzellebene bestätigen. Eine kürzlich erschienene Arbeit auf Einzelzellebene zeigte ebenfalls das Vorliegen einer klonalen T-Zell-Population in LyP, wobei in dieser Studie an 4 Patienten die klonale Population in den CD30-negativen Zellen ausgemacht wurde [21].
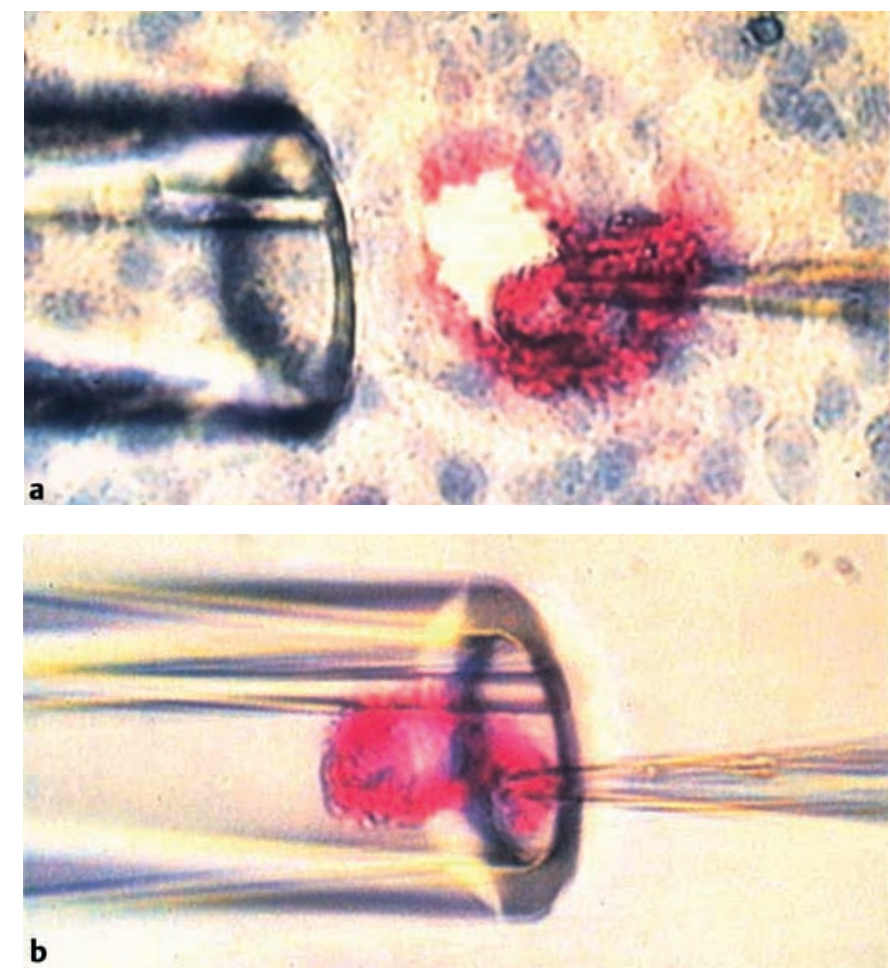

Abb. 3 Vorgang der mechanischen Mikropräparation von CD30-positiven Einzelzellen. a Vorsichtiges Freipräparieren der Zelle mit spitzer Glaskapillare. b Überführen der Einzelzelle in offene Glaskapillare.

Somit konnte gezeigt werden, dass der chronische Verlauf der LyP mit den für diese Erkrankung charakteristischen rezidivierenden, spontanheilenden Hautläsionen auf die Expansion und Regression eines identischen T-Zell-Klons zurückzuführen ist. Damit erfüllt die LyP trotz ihres benignen Verlaufs neben dem malignen histologischen Zellbild ein weiteres klassisches Malignitätskriterium, nämlich das der Monoklonalität, und nimmt hierdurch eine Sonderstellung in der Tumorbiologie ein. Diesen Befunden Rechnung tragend, wird die LyP heutzutage als niedrigmalignes T-Zell-Lymphom mit einer 10-Jahres-Überlebensrate von $100 \%$ gesehen und ist als solches sowohl in der EORTCals auch in der WHO-Klassifikation der Lymphome aufgeführt $[22,23]$.

\section{Pathogenese und Mechanismen der klinischen Regression} und Progression

Der Nachweis, dass es sich bei der LyP um eine monoklonale Erkrankung handelt, hat zwar einiges zur besseren Einordnung und Verständnis dieser Dermatose beigetragen, wirft jedoch weitere Fragen auf:

1. Wie und wo entsteht der Klon und wo verbleibt er in den teilweise monatelangen Phasen der klinischen Remission?

2. Welche Mechanismen führen zur Regression des Klons und welche lassen den Klon der benignen LyP in ein malignes Lymphom übergehen?

Das derzeit gültige Konzept der Entstehung eines malignen Lymphoms sieht eine initiale Tranformation eines einzelnen Lymphozyten vor, die vermutlich nach Akkumulation weiterer Muta- 
tionen in eine unkontrollierte klonale Proliferation des betroffenen Lymphozyten mündet [24]. Prädisponierende oder ursächliche Faktoren hierfür konnten bislang nicht eindeutig identifiziert werden. Verschiedene Faktoren, die eine chronische Antigenstimulation als Folge haben, werden jedoch postuliert [25]. Diese maligne Transformation kann in jedem Reifungsstadium des Lymphozyten erfolgen. Im Falle der extranodalen Lymphome wurde bisher angenommen, dass die maligne Transformation direkt im jeweiligen Manifestationsort, im Falle der kutanen Lymphome also in der Haut, erfolgt. Aktuelle Daten zeigen, dass die initiale Transformation bei der LyP möglicherweise bereits im Knochenmark erfolgen könnte [26].

Dieses für kutane Lymphome allgemein gültige pathogenetische Konzept muss prinzipiell auch für die Pathogenese der LyP angenommen werden.

Untersuchungen zur Klärung des für die LyP charakteristischen Phänomens der spontanen Regression fokussierten auf das bei der LyP exprimierte Oberflächenantigen CD30 und die Interaktion mit seinem Liganden CD30L. Hier konnte eine deutliche Überexpression des CD30-Liganden (CD30L) in abheilenden Läsionen im Vergleich zu persistierenden Läsionen gezeigt werden, eine Beobachtung, die für das Phämomen der spontanen Regression in LyP verantwortlich sein könnte [27]. Die bei LyP bis zu monatelangen Phasen der klinischen Erscheinungsfreiheit vor erneuter klinischer Manifestation weisen auf eine klinisch stumme Persistenz des T-Zell-Klons hin [14,20]. Zu den möglichen Kompartimenten, in denen der Zellklon persistieren könnte, ist neben Haut, Blut und Lymphknoten ebenfalls das Knochenmark anzuführen [26]. Auch sind die Faktoren, die zur erneuten klinischen Manifestation des Zellklons führen, unbekannt. Das in Hautläsionen der LyP ausgeprägt vorhandene reaktive lymphozytäre Infiltrat weist möglicherweise darauf hin, dass die körpereigene zellmediierte Immunabwehr über längere Zeit in der Lage ist, das Zellwachstum bei der LyP zu kontrollieren [28]. Eine eindeutige Korrelation des Rezidivierens der LyP mit Phasen der Immunsuppression konnte jedoch nicht belegt werden.

In $10-20 \%$ der Patienten mit LyP kommt es zumeist nach jahrelangem Verlauf zur Manifestation von malignen Lymphomen, wobei in den meisten Fällen ein gemeinsamer Zellklon in LyP und malignem Lymphom gefunden werden konnte. Die Aufklärung der Mechanismen für diese weitere Transformation bzw. Progression der klinisch „benignen“ klonalen Zellproliferation (LyP) in ein malignes Lymphom ist Gegenstand einiger Studien. Dabei konnte an einer humanen T-Zell-Lymphomzelllinie, die aus einem CD30-positiven T-Zell-Lymphom eines Patienten mit LyP gewonnen wurde, eine Deletion im Gen des Transforming growth factor- $\beta$ (TGF- $\beta$ )-Rezeptors gezeigt werden. Diese Deletion ermöglicht den Zellen des malignen Lymphoms, vermutlich im Gegensatz zum Zellklon der LyP, sich der Wachstumskontrolle durch Transforming growth factor- $\beta$ (TGF- $\beta$ ) zu entziehen [29]. Ferner könnte die Resistenz gegenüber einer CD30-mediierten Wachstumsinhibition eine Rolle für die Progression spielen $[30,31]$. Eine weitere Studie konnte eine vermehrte Fascin-Expression in LyP-Läsionen von Patienten mit assoziiertem systemischen Lymphom zeigen, so dass die Expression von Fascin in LyP als Marker für Progression postuliert wurde [32].
Mehrschritt-Tumorgenese der Lymphome am Beispiel der lymphomatoiden Papulose

All diese die LyP betreffenden Daten belegen beispielhaft das gültige Konzept der Mehrschritt-Tumorgenese. Das oder die initial transformatorischen Ereignisse bei der LyP führen zwar zu monoklonaler Zellexpansion einhergehend mit Zellatypie, reichen aber vermutlich nicht aus, um sich der Kontrolle des Immunsystems entziehen zu können. Erst durch Akkumulation weiterer Mutationen scheint es zur Progression in ein malignes Lymphom mit unkontrolliertem Zellwachstum zu kommen. Dabei ist die Wahrscheinlichkeit diese Mutationen zu erwerben, bedingt durch die deutlich verlängerte Lebensdauer des Zellklons bei der LyP, im Vergleich zu normalen Lymphozyten erhöht.

Eine der LyP vergleichbare Konstellation findet sich bei der MGUS ( = monoclonal gammopathy of undetermined significance), eine Erkrankung, die bis zu 1\% der Normalbevölkerung über dem 50. Lebensjahr betrifft. Trotz jahrzehntelangem asymptomatischen Verlauf wird nur bei einem kleinen Teil dieser Patienten der Übergang in ein malignes Plasmozytom beobachtet [33].

Für die Mehrschritt-Tumorgenese spricht ferner die Beobachtung, dass sich in Patienten mit Auftreten mehrerer morphologisch unterschiedlicher Lymphome, in den meisten Fällen trotz unterschiedlichem histologischen und immunhistologischen Phänotyp, mittels PCR-Analyse ein gemeinsamer Ursprung von einer einzigen transformierten Zelle nachweisen lässt $[12,15,34]$. Die Koinzidenz meherer Lymphome ausgehend von zwei unterschiedlichen, transformierten Zellen ist dagegen eine Rarität [35].

\section{Literatur}

${ }^{1}$ Macauly WL. Lymphomatoid papulosis. A continuing self-healing eruption, clinically benign - histologically malignant. Arch Dermatol 1968; 97: 23-30

2 Wang HH, Lach L, Kadin ME. Epidemiology of lymphomatoid papulosis. Cancer 1992; 70: 2951 - 2957

${ }^{3}$ Bekkenk MW, Geelen FA, Vader PC et al. Primary and secondary cutaneous $\mathrm{CD} 30(+)$ lymphoproliferative disorders: a report from the Dutch Cutaneous Lymphoma Group on the long-term follow-up data of 219 patients and guidelines for diagnosis and treatment. Blood 2000; 95: $3653-3661$

${ }^{4}$ Willemze R, Beljaards RC. Spectrum of primary cutaneous CD30 (Ki1)-positive lymphoproliferative disorders. A proposal for classification and guidelines for management and treatment. J Am Acad Dermatol 1993; $28: 973-980$

${ }^{5}$ El Shabrawi-Caelen L, Kerl H, Cerroni L. Lymphomatoid papulosis: reappraisal of clinicopathologic presentation and classification into subtypes A, B, and C. Arch Dermatol 2004; 140: 441 - 447

${ }^{6}$ Burg G, Hoffmann-Fezer G, Nikolowski J, Schmoeckel C, Braun-Falco O, Stunkel K. Lymphomatoid papulosis: a cutaneous T-cell pseudolymphoma. Acta Derm Venereol 1981; 61: 491 - 496

7 Ploysangam T, Breneman DL, Mutasim DF. Cutaneous pseudolymphomas. J Am Acad Dermatol 1998; 38: 877-895

8 Wood GS, Strickler JG, Deneau DG, Egbert B, Warnke RA. Lymphomatoid papulosis expresses immunophenotypes associated with $T$ cell lymphoma but not inflammation. J Am Acad Dermatol 1986; 15: $444-458$

${ }^{9}$ Kaudewitz P, Stein H, Plewig G et al. Hodgkin's disease followed by lymphomatoid papulosis. Immunophenotypic evidence for a close 
relationship between lymphomatoid papulosis and Hodgkin's disease. J Am Acad Dermatol 1990; 22: 999 - 1006

${ }^{10}$ Kaudewitz P, Herbst H, Anagnostopoulos I, Eckert F, Braun-Falco O, Stein H. Lymphomatoid papulosis followed by large-cell lymphoma: immunophenotypical and genotypical analysis. Br J Dermatol 1991; 124: 465 - 469

${ }^{11}$ Beljaards RC, Willemze R. The prognosis of patients with lymphomatoid papulosis associated with malignant lymphomas. Br J Dermatol 1992; 126: $596-602$

${ }^{12}$ Davis TH, Morton CC, Miller-Cassman R, Balk SP, Kadin ME. Hodgkin's disease, lymphomatoid papulosis, and cutaneous T-cell lymphoma derived from a common T-cell clone. N Engl J Med 1992; 326: 1115 1122

${ }^{13}$ Wood GS, Crooks CF, Uluer AZ. Lymphomatoid papulosis and associated cutaneous lymphoproliferative disorders exhibit a common clonal origin. J Invest Dermatol 1995; 105: 51 - 55

${ }^{14}$ Chott A, Vonderheid EC, Olbricht S, Miao NN, Balk SP, Kadin ME. The dominant $\mathrm{T}$ cell clone is present in multiple regressing skin lesions and associated $\mathrm{T}$ cell lymphomas of patients with lymphomatoid papulosis. J Invest Dermatol 1996; 106: 696-700

${ }^{15}$ Assaf C, Hummel M, Dippel E, Schwartz S, Geilen CC, Harder L, Siebert R, Steinhoff M, Klemke CD, Thiel E, Goerdt S, Stein H, Orfanos CE. Common clonal T-cell origin in a patient with T-prolymphocytic leukaemia and associated cutaneous T-cell lymphomas. Br J Haematol 2003; 120 : $488-491$

${ }^{16}$ Weiss LM, Wood GS, Trela M, Warnke RA, Sklar J. Clonal T-cell populations in lymphomatoid papulosis. Evidence of a lymphoproliferative origin for a clinically benign disease. N Engl J Med 1986; 315: 475 -479

${ }^{17}$ Whittaker S, Smith N, Jones RR, Luzzatto L. Analysis of $\beta, \gamma$, and $\delta$ T-cell receptor genes in lymphomatoid papulosis: cellular basis of two distinct histologic subsets. J Invest Dermatol 1991; 96: 786-791

${ }^{18}$ El Azhary RA, Gibson LE, Kurtin PJ, Pittelkow MR, Muller SA. Lymphomatoid papulosis: a clinical and histopathologic review of 53 cases with leukocyte immunophenotyping, DNA flow cytometry, and T-cell receptor gene rearrangement studies. J Am Acad Dermatol 1994; 30: $210-218$

${ }^{19}$ Kadin ME, Vonderheid EC, Sako D, Clayton LK, Olbricht S. Clonal composition of T cells in lymphomatoid papulosis. Am J Pathol 1987; 126: $13-17$

20 Steinhoff M, Hummel M, Anagnostopoulos I, Kaudewitz P, Seitz V, Assaf C, Sander C, Stein H. Single-cell analysis of CD30+ cells in lymphomatoid papulosis demonstrates a common clonal T-cell origin. Blood 2002; 100: 578 - 584

${ }^{21}$ Gellrich S, Wernicke M, Wilks A, Lukowsky A, Muche JM, Jasch KC, Audring H, Mason D, Sterry W. The cell infiltrate in lymphomatoid papulosis comprises a mixture of polyclonal large atypical cells (CD30-positive) and smaller monoclonal T cells (CD30-negative). J Invest Dermatol 2004; 122: 859-861

${ }^{22}$ Willemze R, Kerl H, Sterry W, Berti E, Cerroni L, Chimenti S et al EORTC classification for primary cutaneous lymphomas: a proposal from the Cutaneous Lymphoma Study Group of the European Organization for Research and Treatment of Cancer. Blood 1997; 90: $354-$ 371

${ }^{23}$ Jaffe ES, Harris NL, Stein H (eds.) et al. Pathology and Genetics: Tumours of Haematopoietic and Lymphoid Tissues. Lyon, France: IARC Press, 2001

24 Dummer R, Willers J, Kamarashev J, Urosevic M, Dobbeling U, Burg G. Pathogenesis of cutaneous lymphomas. Semin Cutan Med Surg 2000; 19: $78-86$

${ }^{25}$ Burg G, Dummer R, Haeffner A, Kempf W, Kadin M. From inflammation to neoplasia: mycosis fungoides evolves from reactive inflammatory conditions (lymphoid infiltrates) transforming into neoplastic plaques and tumors. Arch Dermatol 2001; 137: 949-952

${ }^{26}$ Gniadecki R, Lukowsky A, Rossen K, Madsen HO, Thomsen K, Wulf HC. Bone marrow precursor of extranodal T-cell lymphoma. Blood 2003; 102: $3797-3799$

${ }^{27}$ Mori M, Manuelli C, Pimpinelli N, Mavilia C, Maggi E, Santucci M, Bianchi B, Cappugi P, Giannotti B, Kadin ME. CD30-CD30 ligand interaction in primary cutaneous $\mathrm{CD} 30(+) \mathrm{T}$-cell lymphomas: A clue to the pathophysiology of clinical regression. Blood 1999; 94: 3077-3083

${ }^{28}$ Agnarsson BA, Kadin ME. Host response in lymphomatoid papulosis. Hum Pathol 1989; 20: 747 - 752

${ }^{29}$ Schiemann WP, Pfeifer WM, Levi E, Kadin ME, Lodish HF. A deletion in the gene for transforming growth factor beta type I receptor abolishes growth regulation by transforming growth factor beta in a cutaneous T-cell lymphoma. Blood 1999; 94: 2854-2861

${ }^{30}$ Levi E, Wang Z, Petrogiannis-Haliotis T, Pfeifer WM, Kempf W, Drews R, Kadin ME. Distinct effects of CD30 and Fas signaling in cutaneous anaplastic lymphomas: a possible mechanism for disease progression. J Invest Dermatol 2000; 115: 1034 - 1040

31 Kadin ME, Levi E, Kempf W. Progression of lymphomatoid papulosis to systemic lymphoma is associated with escape from growth inhibition by transforming growth factor-beta and CD30 ligand. Ann N Y Acad Sci 2001; 941: 59-68

32 Kempf W, Levi E, Kamarashev J, Kutzner H, Pfeifer W, PetrogiannisHaliotis T, Burg G, Kadin ME. Fascin expression in CD30-positive cutaneous lymphoproliferative disorders. J Cutan Pathol. 2002; 29: 295 300

33 Kyle RA, Therneau TM, Rajkumar SV, Offord JR, Larson DR, Plevak MF, Melton LJ 3rd. A long-term study of prognosis in monoclonal gammopathy of undetermined significance. N Engl J Med 2002; 346: 564-569

${ }^{34}$ Marafioti T, Hummel M, Anagnostopoulos I, Foss HD, Huhn D, Stein H. Classical Hodgkin's disease and follicular lymphoma originating from the same germinal center B cell. J Clin Oncol. 1999; 17: 3804- 3809

${ }^{35}$ Steinhoff M, Hummel M, Assaf C, Anagnostopoulos I, Treudler R, Geilen CC, Stein H, Orfanos CE. Cutaneous T cell lymphoma and classic Hodgkin lymphoma of the B cell type within a single lymph node: composite lymphoma. J Clin Pathol. 2004; 57: 329-331 